\title{
APRESENTAÇÃO À SEXTA EDIÇÃO DA RFPTD
}

Iniciamos a apresentação da $6^{\mathrm{a}}$ edição da Revista de Finanças Públicas, Tributação e Desenvolvimento, vinculada ao Programa de Pós-Graduação em Direito da UERJ, dedicando o presente número à memória do Professor Ricardo Lobo Torres, brilhante jurista que deixa extensa e valiosa obra para toda comunidade acadêmica.

O Professor Ricardo Lobo Torres, ao longo de sua carreira, abrilhantou a Equipe Editorial da Revista, compondo desde o início a Presidência do Conselho Editorial. Influenciou a formatação e o desenvolvimento da linha editorial, consentânea com o pluralismo e a diversidade que marcam a sociedade brasileira.

Nesta edição, os aspectos valorativos que permeiam o direito tributário são destacados em estudos sobre o poder tributário, justiça tributária, federalismo fiscal, segurança jurídica e o perfil constitucional das contribuições.

A Revista tem como principal objetivo disponibilizar aos leitores artigos que alinham temas sobre direito financeiro e tributário com todo o sistema normativo nacional e internacional, ampliando as possibilidades de pesquisa e investigação científica.

Em breve será realizada a chamada para a $7^{\mathrm{a}}$ edição.

Desejamos a todos uma boa leitura!

\author{
RICARDO LODI RIBEIRO* \\ JULIO CESAR SANTIAGO ${ }^{* *}$ \\ VANESSA BENELLI CORRÊA***
}

\footnotetext{
* Editor Chefe e Diretor da Faculdade de Direito da UERJ

** Editor Adjunto, Doutorando e Mestre em Finanças Públicas, Tributação e Desenvolvimento pela UERJ.

*** Editora Assistente, Doutoranda e Mestre em Finanças Públicas, Tributação e Desenvolvimento pela UERJ.
}

Revista de Finanças Públicas, Tributação e Desenvolvimento - RFPTD, v. 6, n.6, 2018 


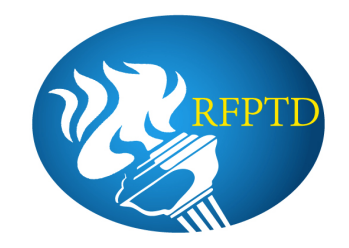

\section{EDITOR CHEFE}

Ricardo Lodi Ribeiro, Universidade do Estado do Rio de Janeiro - UERJ, Brasil

\section{EDITOR ADJUNTO}

Julio Cesar Santiago, Universidade do Estado do Rio de Janeiro - UERJ, Brasil

\section{EDITORA ASSISTENTE}

Vanessa Benelli Corrêa, Universidade do Estado do Rio de Janeiro, Brasil, Brasil

PRESIDENTE DO CONSELHO EDITORIAL (In memoriam)

Ricardo Lobo Torres, Universidade do Estado do Rio de Janeiro - UERJ, Brasil

\section{CONSELHO EDITORIAL}

Humberto Ávila, Universidade Federal do Rio Grande do Sul - UFRS, Brasil

James Marins, Pontifícia Universidade Católica do Paraná - PUC/PR, Brasil

Prof. Dr. José Casalta Nabais, Universidade de Coimbra, Portugal

Luís Eduardo Schoueri, Universidade de São Paulo - USP, Brasil

Marciano Seabra de Godoi, Pontifícia Universidade Católica de Minas Gerais - PUC Minas, Brasil

Pedro M. Herrera Molina, Universidad Complutense de Madrid, Espanha

Ricardo Lodi Ribeiro, Universidade do Estado do Rio de Janeiro - UERJ, Brasil

\section{COMITÊ EXECUTIVO}

Julio Cesar Santiago, Universidade do Estado do Rio de Janeiro - UERJ, Brasil

Vanessa Benelli Corrêa, Universidade do Estado do Rio de Janeiro - UERJ, Brasil

\section{CONSELHO DE PARECERISTAS}

Adilson Rodrigues Pires, Universidade do Estado do Rio de Janeiro, Brasil

André Folloni, Pontifícia Universidade Católica do Paraná, Brasil

André Mendes Moreira, Universidade Federal de Minas Gerais, Brasil, Brasil

Arnaldo Sampaio de Moraes Godoy, Centro Universitário de Brasília, Brasil

Arthur Maria Ferreira Neto, Pontifícia Universidade Católica do Rio Grande do Sul, Brasil

Carlos Alexandre de Azevedo Campos, Universidade doe Estado do Rio de Janeiro, Brasil

Carlos Araújo Leonetti, Universidade Federal de Santa Catarina, Brasil

Revista de Finanças Públicas, Tributação e Desenvolvimento - RFPTD, v. 6, n.6, 2018 


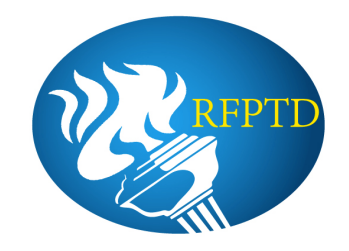

Carlos Eduardo Peralta Montero, Universidad de Costa Rica, Costa Rica

Cristiane Miranda Botelho, Universidade Federal de Minas Gerais, Brasil

Daniela Olímpio Oliveira, Universidade Federal de Lavras, Brasil

Denise Lucena Cavalcante, Universidade Federal do Ceará, Brasil

Elizabete Rosa de Mello, Universidade Federal de Juiz de Fora, Brasil

Fabio Zambitte Ibrahim, Universidade do Estado do Rio de Janeiro, Brasil

Gustavo da Gama Vital de Oliveira, Universidade do Estado do Rio de Janeiro, Brasil

Hugo de Brito Machado Segundo, Universidade Federal do Ceará, Brasil

Igor Mauler Santiago, Universidade Federal de Minas Gerais, Brasil, Brasil

José Eduardo Silvério Ramos, PUC/SP - Pontifícia Universidade Católica de São Paulo, Brasil

José Maria Arruda de Andrade, Universidade de São Paulo, Brasil

James Marins, Pontíficia Universidade Católica do Paraná, Brasil

João Bosco Coelho Pasin, Universidade Plesbiteriana Mackenzie, Brasil

Larissa Rodrigues Laks, Pontifícia Universidade Católica do Rio Grande do Sul, Brasil

Liziane Angelotti Meira, Universidade Católica de Brasília, Brasil

Ludmila Mara Monteiro de Oliveira, Universidade Federal de Minas Gerais, Brasil, Brasil

Luís Cesar Queiroz, Universidade do Estado do Rio de Janeiro, Brasil

Luís Eduardo Schoueri, Universidade de São Paulo, Brasil

Marciano Buffon, Universidade do Vale do Rio dos Sinos, Brasil

Marciano Seabra de Godoi, Pontifícia Universidade Católica de Minas Gerais, Brasil

Marcus Lívio Gomes, Universidade do Estado do Rio de Janeiro, Brasil

Mariana Barboza Baeta Neves, Pontifícia Universidade Católica de São Paulo, Brasil

Marta Villar Ezcurra, Universidad de San Pablo, Espanha

Paulo Caliendo, Pontifícia Universidade Católica do Rio Grande do Sul, Brasil

Regina Helena Costa, Pontifícia Universidade Católica de São Paulo, Brasil

Robson Maia Lins, Pontifícia Universidade Católica de São Paulo, Brasil

Sérgio André Rocha, Universidade do Estado do Rio de Janeiro, Brasil

Sérgio Vasques, Universidade de Lisboa, Portugal

Vanessa Benelli Corrêa, Universidade do Estado do Rio de Janeiro, Brasil, Brasil 